\title{
A Paradigm for Object-oriented Conceptual Modeling of Optical Networks*
}

\author{
Sudip Misra ${ }^{1}$, Subhas Chandra Misra ${ }^{2}$ and Isaac Woungang ${ }^{3}$ \\ ${ }^{1}$ Department of Computer Science, Yale University, CT, USA \\ ${ }^{2}$ SuperNet Solutions, Toronto, ON, Canada \\ ${ }^{3}$ School of Computer Science, Ryerson University, Toronto, ON, Canada
}

\begin{abstract}
We present a conceptual framework for modeling optical networks and the solutions to its associated problems. The framework is targeted towards a large-scale initiative by us for developing a modeling language that can help with conceptual modeling of typical optical network design problems. While there are pieces of literature available that describe modeling languages for other application areas, there are no specific languages for use in networking problems in general. Our work is an important step forward to address this defficiency. Our proposed framework takes the already existing modeling language notations and extends them to support the modeling of optical networks.
\end{abstract}

Keywords: object-oriented design, conceptual modeling, optical networks, distributed systems, UML-based profile.

\section{Introduction}

In this paper we present an UML-based framework to support modeling optical networking problems. Our efforts are targeted towards a larger scale initiative for providing an UML Profile for modeling Optical Networks. The Profile is based on an extension of UML 1.5. Although UML is considered by many professionals as a de facto tool to aid in system modeling, the current versions of UML do not specify methodologies, modeling elements and tools to address problems specific to networking. The UML Profile we are developing for use in optical networks enables one to model fundamental concepts in network communications, optical network hardware, structure of protocols and optical network component interactions.

Other initiatives with UML Profiles address modeling issues associated with application domains such as Quality of Service in Distributed Systems [1,2], Business Modeling [4], Fault Tolerance [5], CORBA [6], Testing [7] and Schedulability, Performance and Time [8] and Agentoriented Software [9].

Although there have been UML Profiles developed for use in other application areas, there are no UML-based profiles even for use in modeling the fundamental networking problems. Since optical networks are one of the most promising modern day communication technologies, we focused our work in this area.

In this paper, we illustrate some fundamental optical networking concepts $[11,12,13]$ using our modeling approach, however the approach is extensible. At the outset we should disclaim that the work is not a robust approach to consider modeling all possible optical networking scenarios. However, we do believe that our paper can definitely help the reader in extending the concepts and methodologies we discuss in this paper for use in specific problems.

One of the important advantages of optical networks is that they provide very high carrier frequency of light and minimum dispersion of signal. Essentially, light is an ideal carrier for transfer of information because communication

\footnotetext{
${ }^{*}$ A preliminary version of this paper appeared in the Proceedings of the $8^{\text {th }}$ International Conference on Advanced Communications Technologies, IEEE Communications Society, Phoenix Park, Korea, February 20-22, 2006.
} 
through light has an advantage of minimizing the interaction between light rays with different wavelengths. This allows having many independent light rays with different wavelengths that can pass through one fibre. This multiplies the capacity of the fibre to the number of wavelengths which are used for communication purposes. Also, the advantage of optical data transmission is the ability to operate with light signal fully in the optical range, avoiding optical to electric (OE) and electric to optical (EO) conversions, which may slow the transfer rate and overall capacity of optical networks. The simplest of the optical network hardware, which operates with the signal fully in the optical domain, is coupler, which allows adding/dropping one wavelength to/from the optical signal.

For constructing and modeling of optical networks [10, 13], many factors usually should be taken into account. As the optical network hardware is quite expensive, economical considerations must be taken into account while building optical networks. A high-level view of actions required for the development of optical networks are shown in Figures 1-3.

Therefore, the following marketing management and technology management phases are used: an advantage assessment of results of introduction, an estimation of the market demands and forecasting, selecting network hardware and network software, customization, modeling and introduction. The following strategies can be taken into consideration while developing an optical network:

- Construct at once all network on available demand and forecasts, and

- Construct a backbone with extensions on demands.

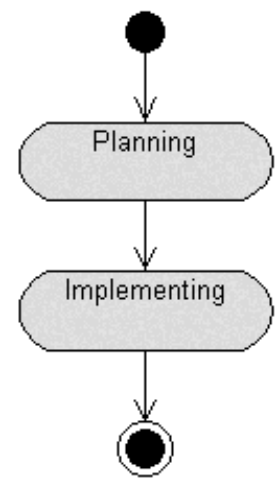

Fig. 1. High-level actions for developing an optical network.

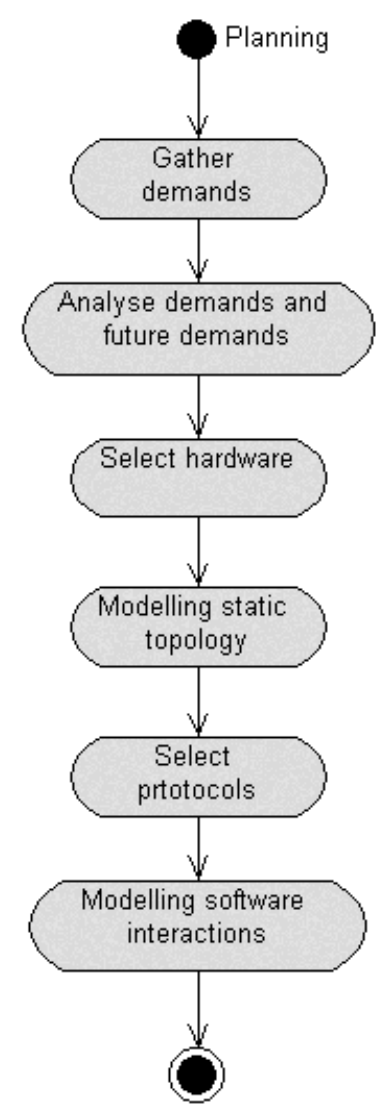

Fig. 2. Optical network planning actions.

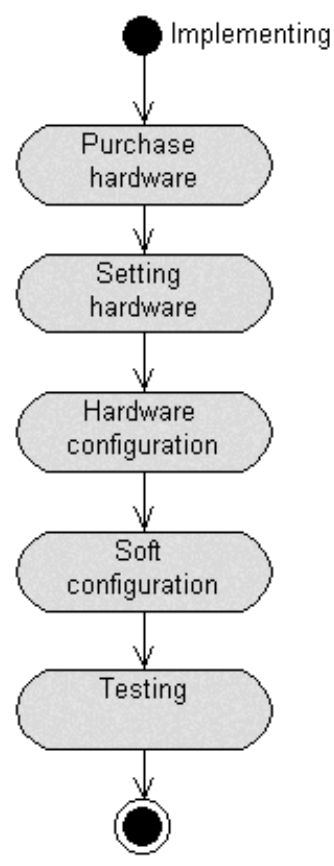

Fig. 3. Optical network implementation actions. 
The necessary quality of service $Q o S$ must be taken into account in the planning process. Thus choosing appropriate protection schemes such as $1: 1$ or $1: N$ must be considered while modeling optical networks, depending on the appropriate $Q o S$.

The main problems that need to be solved for modeling optical networks are the Physical Topology Design, Virtual Topology Design and Dynamic RWA Problem. Physical Topology Design is used after a choice of technologies and strategy of the development have been made. The physical topology greatly depends on the routing of lightpaths and wavelength assignment strategy [10].

\section{Overall Design Rationale}

The development of optical networks is quite complex, and its successful modeling requires thorough understanding of many components, definitions and technologies. We assume that the reader is familiar with the area of optical networks. But before presenting the details of our work, we just review some of the concepts that might help in the understanding of this paper.

Computer networks are designed to provide information exchange between participants. A network acts as an "information mediator". Every network can be considered as the mediator between an information source and a recipient. Thus a network can be viewed as an interoperation of many information mediators. In optical networks, the principal mediators are the fibres and $O X C$ s. There is also specialized hardware meant for use in optical networks, but most of them serve as information mediators. Information in Optical Networks can be classified as either - "transfer information", or "controlling information".

The following terminology is used in the profile. A modifier is an object which can change information. A mediator serves the role of an intermediate communicator. First it receives information then it can change it, then it sends it to next recipient(s). So mediator is a recipient, a source and maybe even a modifier. A network object is any object, which is a part of the computer network. A network communicator is a network object capable of sending and receiving information. The rest of the terminology used in the profile is standard in Optical Communication literature and is not explained here in further detail.

\section{The UML-based Modeling Framework: Towards an UML-profile}

The Profile itself describes main purpose concepts and classes. The Profile was developed in the form of object-oriented analysis models of the optical networks data domain. Static models show the main concepts used in optical networks and their relations. Network protocols described in this profile are basically Internet protocols. So this profile shows how to model optical networks concepts from the IP from the optical networks point of view.

The structure of this profile is simple enough for use in real applications. As mentioned earlier, the approach can be extended and tailored to satisfy the needs of the specific problem being analyzed. We analyze the data domain first.

Structure of the profile: The profile consists of the corresponding packages. Every package contains classes, which can be used in modeling of optical networks. They define and describe data domain of communications and optical networks. All of them are model classes, except Fiber, which is a LinkObject stereotype. So this LinkObject association stereotype can provide connections between optical network hardware.

The Profile consists of different packages. The top-level package is the MoON Profile package (see Figure 4). The model elements of the Profile are stored in this package. The MoON Profile package contains the Communication and Optical Network packages, as shown in Figure 5.

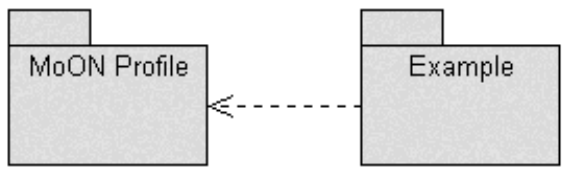

Fig. 4. Top package. 


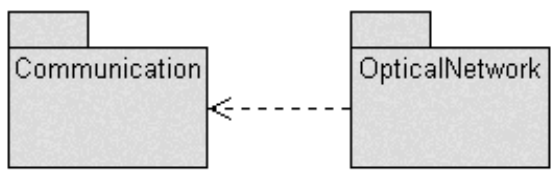

Fig. 5. MoON Profile package.

Communication Package: The Communication Package contains the GeneralCommunication and NetworkCommunication packages as shown in Figure 6. Communication Package contains elements, which describe the general network communication roles and protocols.

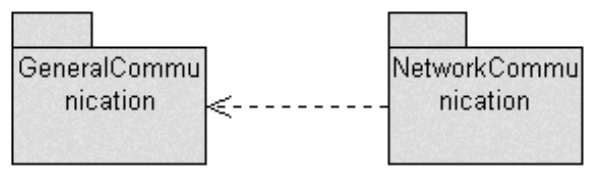

Fig. 6. Communication Package.

General Communication Package: The General Communication Package contains GeneralCommunicationRoles and GeneralProtocol Packages, as shown in Figure 7. The detalis of the GeneralCommunicationRoles Package are explained in Figures 8-10. The Network Communication Package depends on the General Communication Package. This package describes the general communications that takes place in all IP networks.

Communicator and information are basic concepts of information exchange. The Figures 810 describing the different communication concepts in the GeneralCommunicationRoles Package are self-explanatory. Communicator is an object that is capable of sending or receiving information. Basic concepts of information exchange are the source, recipient and modifier. Source and recipient are communicators capable of sending and receiving information. Modifier can change information in some way. Information exchange can be unidirectional, bidirectional or multicast. Every mediator may consist of at least one recipient, and one source, and it can also contain modifier(s). Every me-

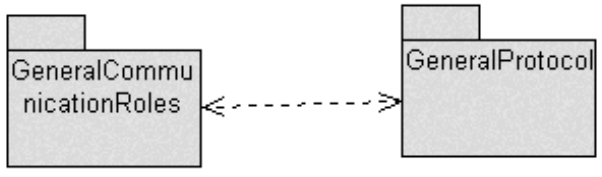

Fig. 7. GeneralCommunication Package. diator can also be an owner of other mediators. Message is a special case of information or its part sent from source to recipient(s) as a single block of information.

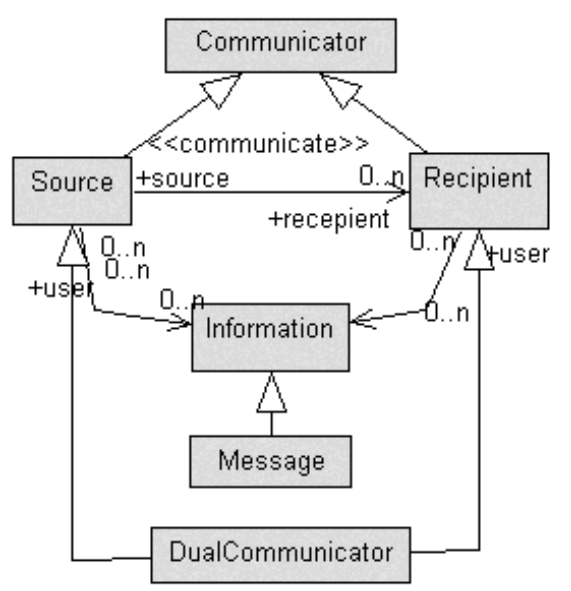

Fig. 8. GeneralCommunicationRoles package. Main communication concepts and relations.

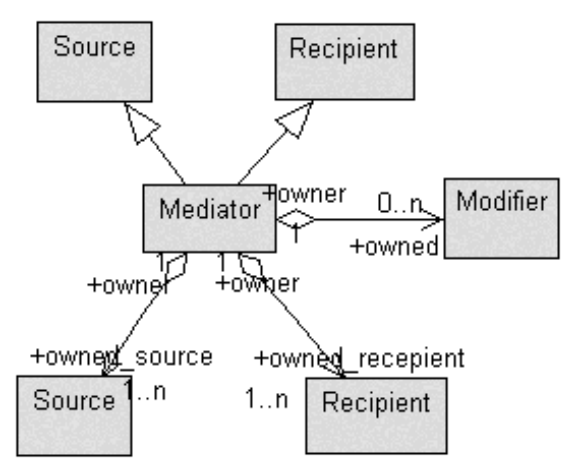

Fig. 9. GeneralCommunicationRoles package. Mediator.

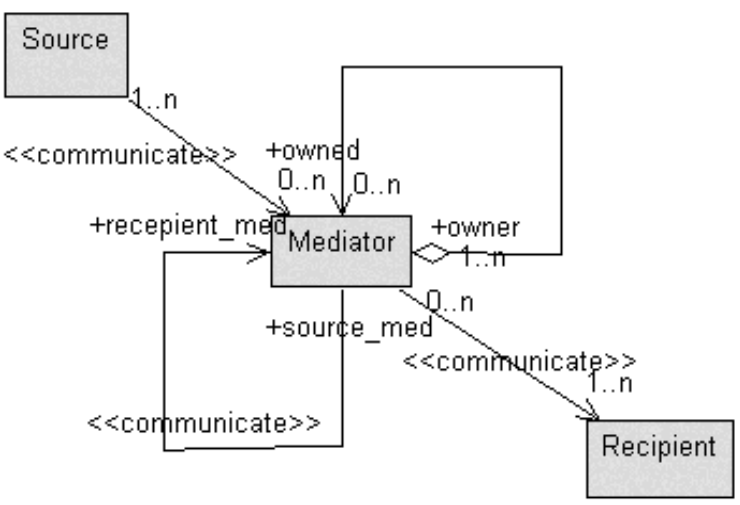

Fig. 10. GeneralCommunicationRoles package. Mediator communication and hierarchy. 


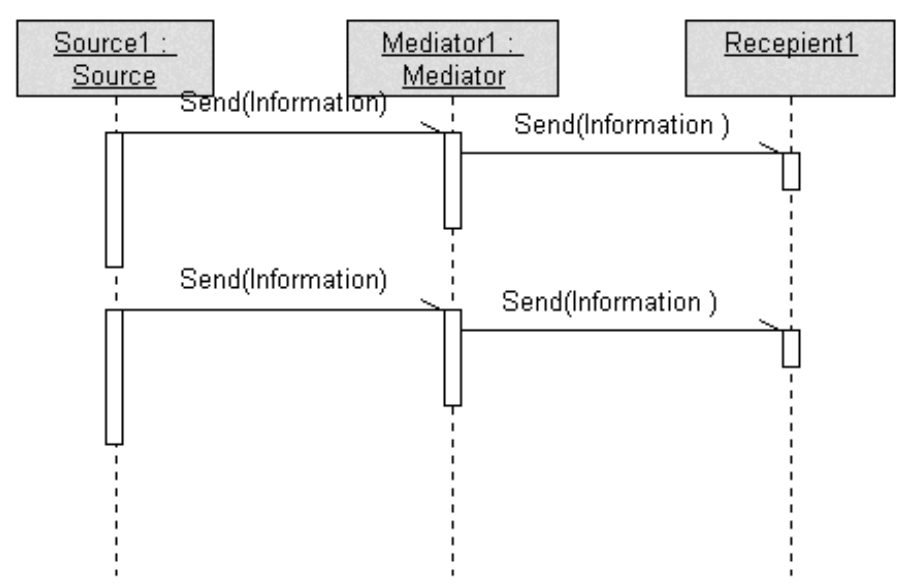

Fig. 11. GeneralCommunication package. Unidirectional communication.

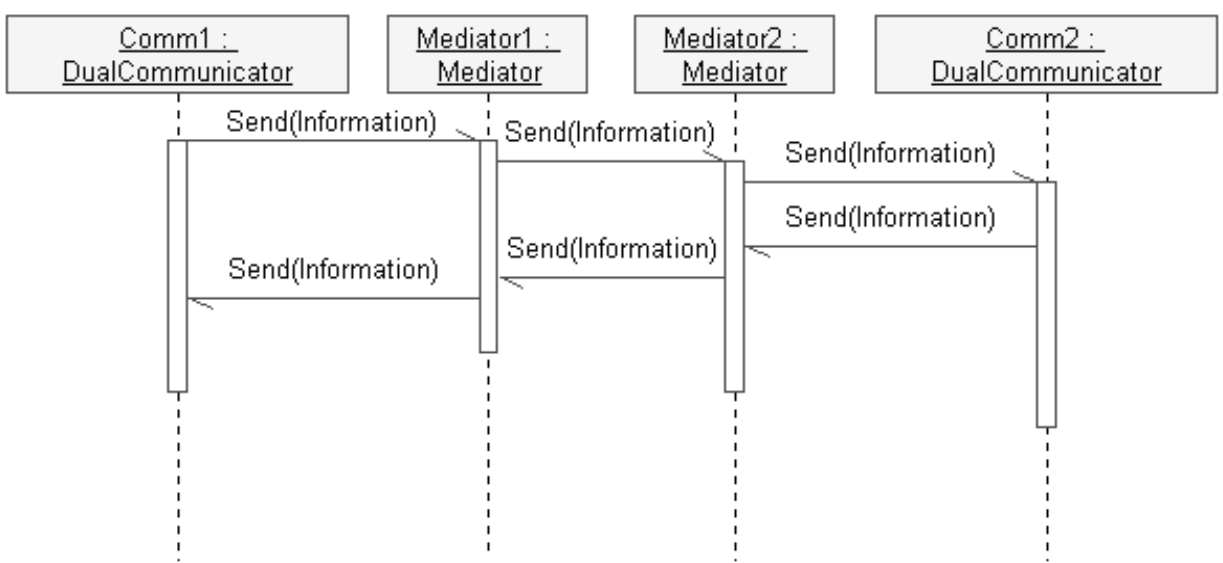

Fig. 12. GeneralCommunication package. Dual directional communication.

The sequence diagrams in Figures 11-12 show the general communication behaviour between communication objects. Figure 11 shows unidirectional communication through one mediator, whereas Figure 12 shows bi-directional communication through two mediators.

Communication protocols are used for the purpose of encoding and decoding of the information by specified set of communication rules. Protocol defines certain communication rules in such a way that communicators can understand each other.

Each communicator contains a protocol stack that contains protocol layers. Each protocol layer implements a specific protocol. Several protocol layers form a protocol stack. Layers in protocol stack can communicate with each other. Usually, the source of information passes through sender's protocol stack. In the protocol stack, it passes through a stack of protocol layers. Each layer formats or transforms information using protocol rules. So the information could be sent through some medium or mediator. The recipient receives information and passes it through its protocol stack, but in reverse direction, so that the information can be transformed to its original state. Figure 13 shows the communicator protocol stack structure in the GeneralProtocol Package, whereas Figure 14 shows an UML sequence diagram depicting the protocol layer interactions while sending information from source to recipient.

NetworkCommunication Package: The NetworkCommunication package contains NetworkCommunicationRoles and NetworkProtocol Packages, as shown in Figure 15. 


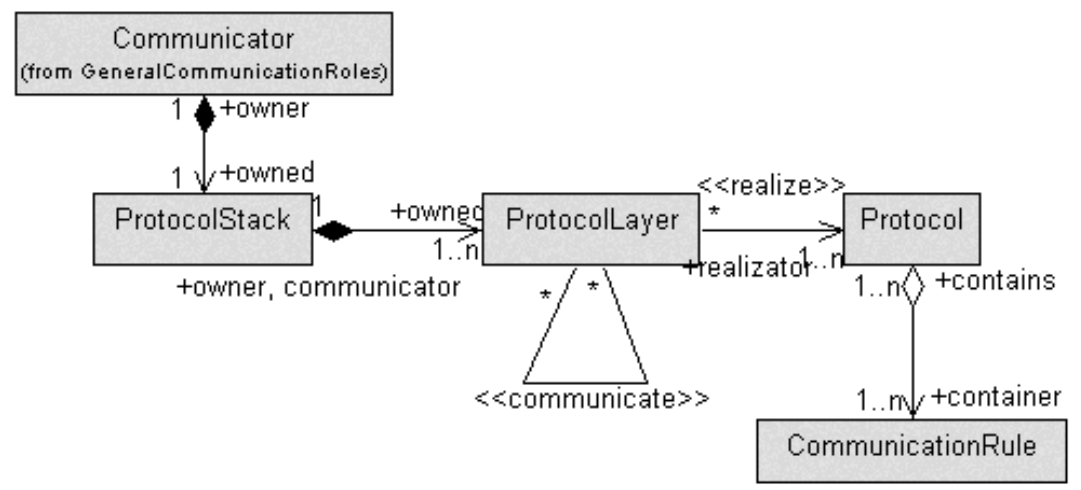

Fig. 13. GeneralProtocol Package. Communicator protocol stack structure.

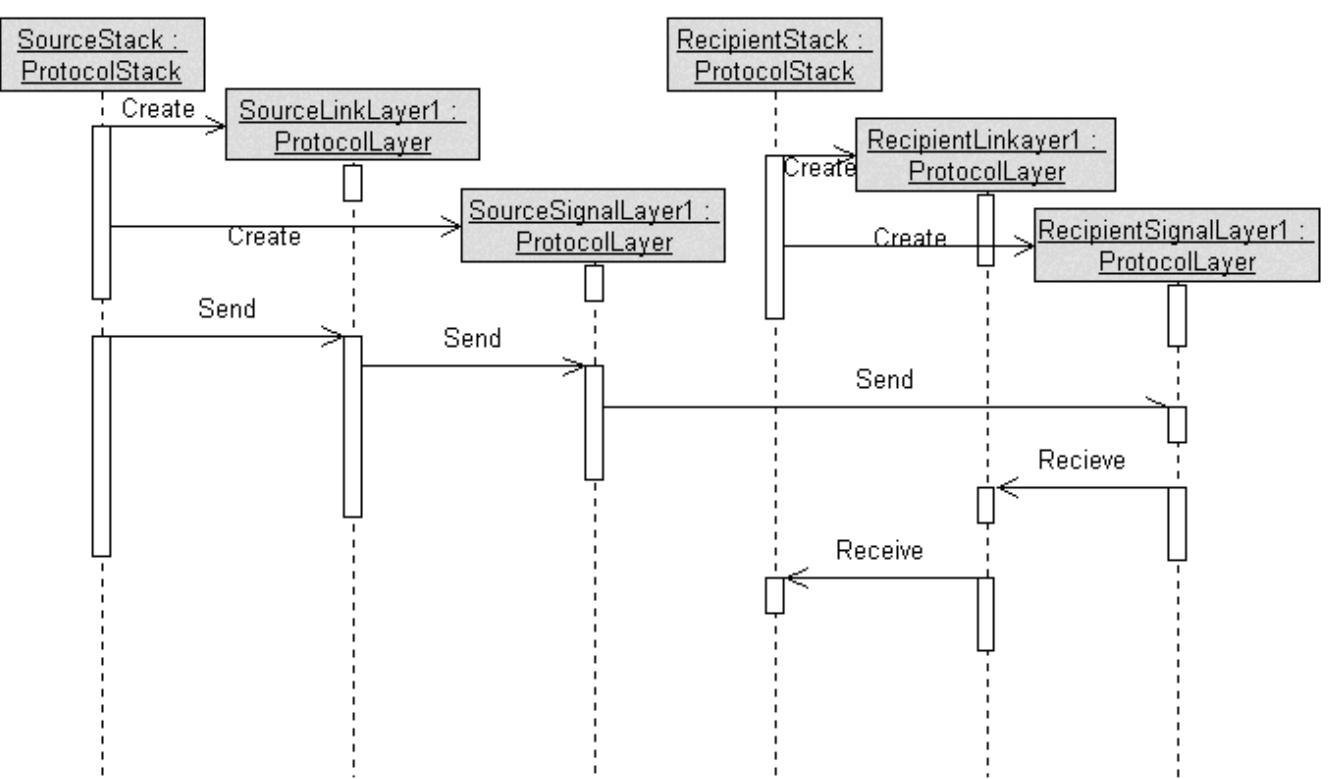

Fig. 14. Protocol stack and protocol layer interactions while sending information from source to recipient.

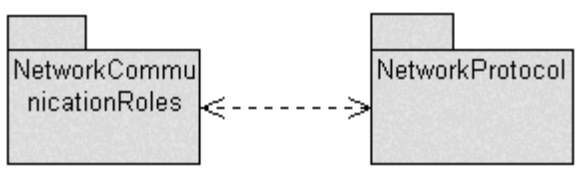

Fig. 15. NetworkCommunication Package.

The NetworkCommunicationRoles Package is shown in Figures 16-17. As can be seen in these figures, the NetworkCommunicationRoles package defines NetworkCommunicator that is a descendant of Communicator and NetworkObject. It also defines Client, Server and Peer classes, which are descendants of NetworkCommunicator classes. Network communicator is a special case of a generic communicator - so it can per- form communication with other communicators and is a network object. Therefore, it can be included in the network. Network communicator is a hardware or software that can send or receive information to other communicators. Like every communicator, it implements a protocol stack. Specific instances of network communicators are Client, Server and Peer.

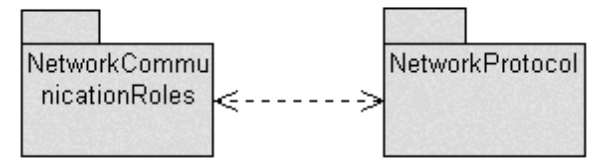

Fig. 16. NetworkCommunicationRoles package. Network Communicator definition. 


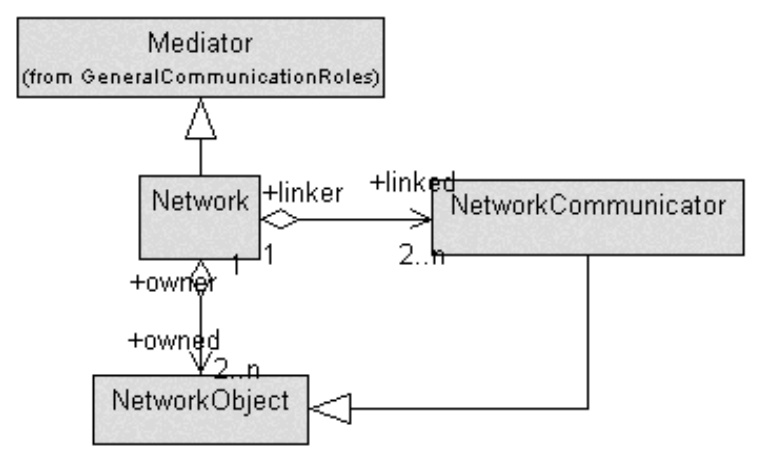

Fig. 17. NetworkCommunicationRoles package. Network definition.

A network can be implemented using one of two basic architectures: client-server or peerto-peer. In client-server architecture, some network communicator (server) provides services to other network communicators (clients). In peer-to-peer architecture, every communicator has almost equal capabilities. Network communicator implements computer network pro$\operatorname{tocol}(\mathrm{s})$.

A network is a mediator. Therefore a network can be represented using a class, which must contain a minimum of two NetworkCommunicators. Then network consists of NetworkObjects. Network communicators and auxiliary network objects can group network objects.

Network protocol package contains main definitions of network protocols.
Figure 18 shows the conceptual class diagram showing the different network protocol types as classes. Computer network protocols can be classified either as control protocols or data transfer protocols. Control protocols are auxiliary protocols that are used for network management purposes. Examples of control protocols are ARP, ICMP, IGMP and other. Data transfer protocols are used for direct transferring of the information.

Figure 19 shows the different class of Internet protocols classified as different packages. Network protocols can be divided by the items for which they specify communication rules. Hardware protocols specify rules of communication at the hardware level, such as shape

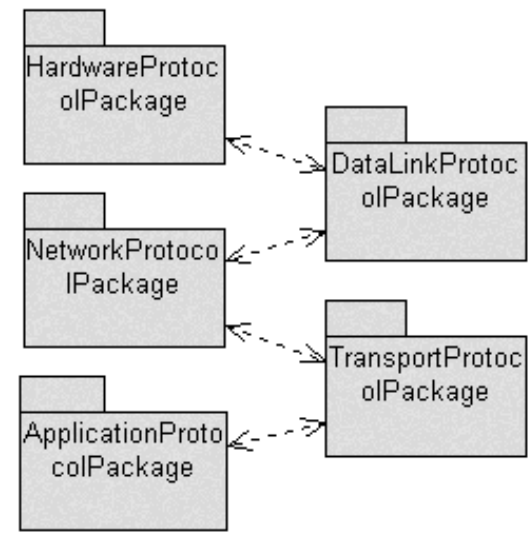

Fig. 19. Internet protocol layers.

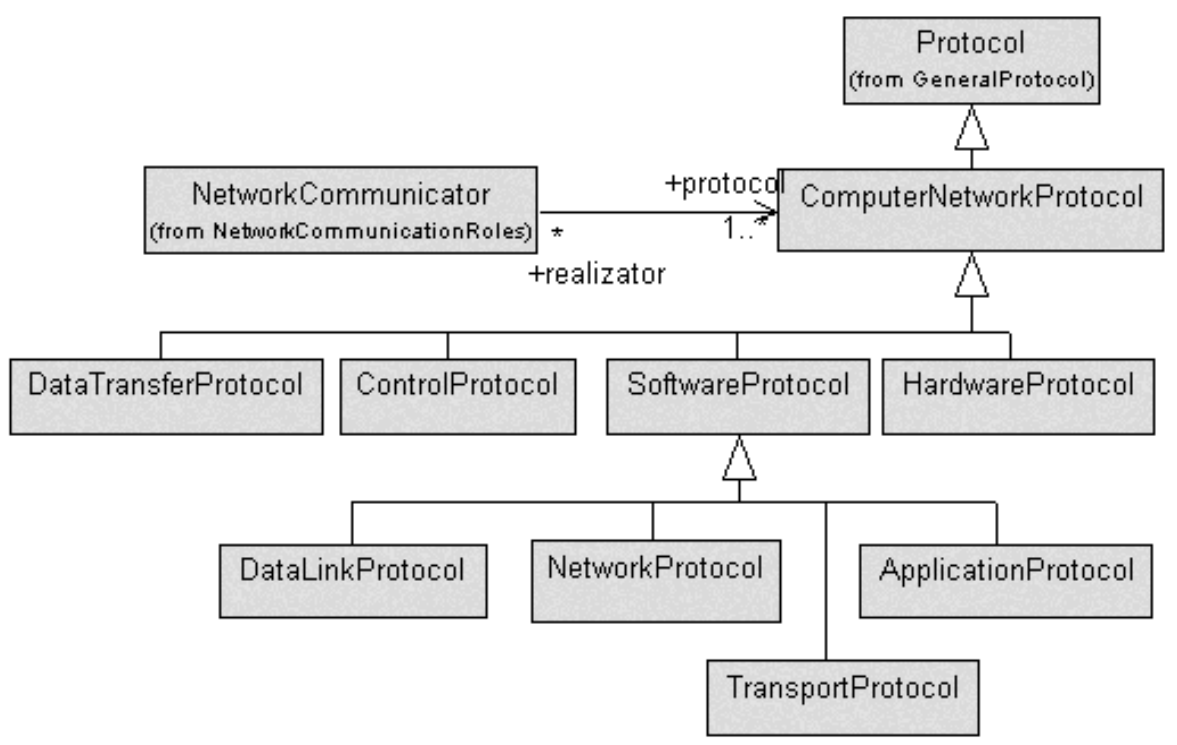

Fig. 18. Computer network protocol types. 


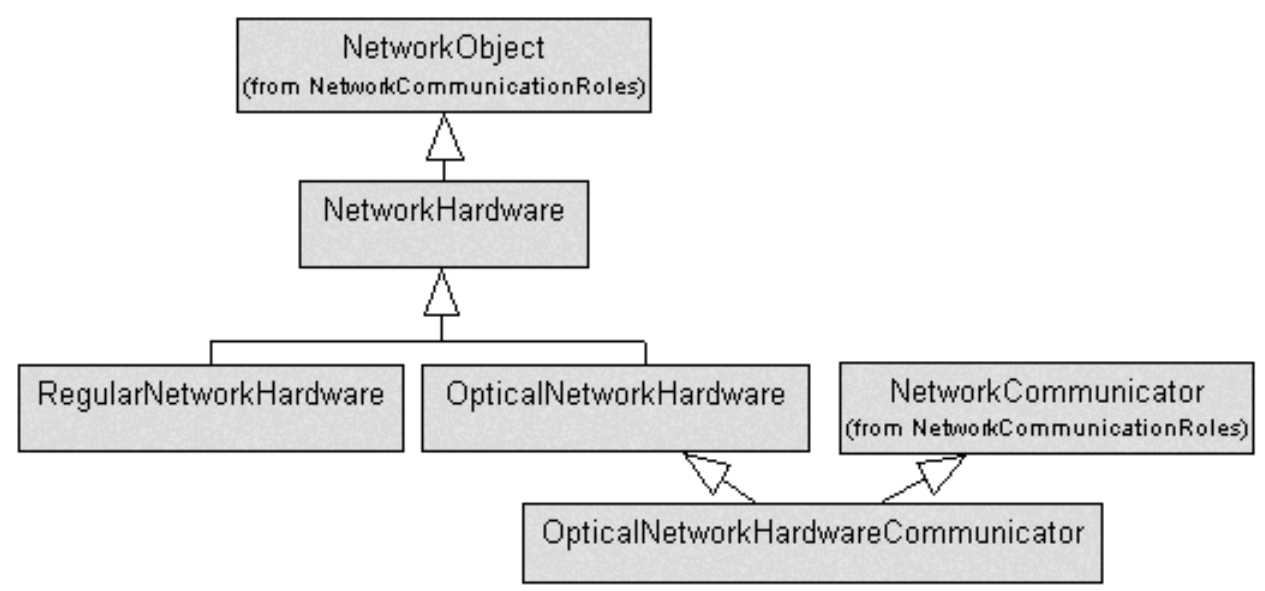

Fig. 20. Optical Network Hardware and Optical Network Hardware Communicator.

of the electrical signal, its amplitude and other characteristics of the signal. Software protocols specify communication rules at software level. Software level indicates the level of CPU code instructions, which are executed by CPU.

Optical Network Package: The Optical Network Package contains the Optical Hardware Package. Figure 20 shows an UML class diagram depicting NetworkHardware, RegularNetworkHarware, OpticalNetworkHardware, and OpticalNetworkHardwareCommunicator. The class names indicate their roles/purpose and are self-explanatory.

Fiber stereotype (shown in Figure 21) allows linking of OpticalNetworkHardwareCommunicator classes. It simplifies designing of optical network models by using UML associations. It must have two ends of the links. It must interconnect OpticalNetworkHardwareCommunicators. It has one String tag - modetype. Its value must be "single", "multi" or "empty". If its value is empty, then it is considered by default that the fiber is multimode.

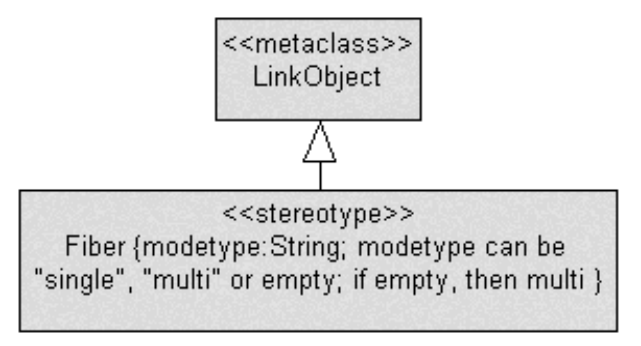

Fig. 21. Fiber stereotype.

\section{Conclusion}

In this paper we provided an UML-based conceptual framework to aid in modeling optical networking systems and in solving their associated problems. The work is targeted towards developing an UML-based profile to aid in modeling optical networks. The work is a significant contribution to the networking community as the complexity of optical networks demands great efforts to analyze, model and standardize the solution approaches to various problems commonly occurring in the area of optical networks.

\section{References}

[1] J. O. AAgedal AND E. F. ECKLUnd JR., Modeling $Q o S$ : Towards a UML Profile, Proceedings of UML 2002, Dresden, Germany, September 20 - October 4, 2002, LNCS 2460, pp. 275-289.

[2] J. O. AAgEDAL, Quality of Service Support in Development of Distributed Systems, PhD Thesis, Department of Informatics, Faculty of Mathematics and Natural Sciences, University of Oslo, 2001.

[3] D. Awduche et AL., Overview and Principles of Internet Traffic Engineering, RFC 3272, IETF.

[4] S. Johnston, Rational UML Profile for Business Modeling, June 2004,

http://www-106.ibm.com/developerworks /rational/library/5167.html?ca=dnp-327

[5] OMG, UML Profile for Modeling Quality of Service and Fault Tolerence Characteristics and Mechanisms, Request for Proposal, ad/02-01-07, January, 2002. 
[6] OMG, UML Profile for CORBA, Version 1.0, http: //www .omg.org/docs/formal 102-04-01.pdf

[7] OMG, UML 2.0 Testing Profile Specification, September 2003,

http: //www .omg.org/docs/ptc

104-04-02.pdf

[8] OMG, UML Profile for Schedulability, Performance and Time, Version 1.0, September 2003, http: //www.omg.org/docs/formal 103-09-01.pdf

[9] G. Wagner, A UML Profile for Agent-oriented Modeling, Technical Report, I\&T Department, Eindhoven University of Technology, 2002, The Netherlands. http://tmitwww.tm.tue.nl/staff/gwagner/

[10] G. N. Rouskas, H. G. Perros, A Tutorial on Optical Networks, Networking 2002 Tutorials, LNCS 2497, pp. 155-193, 2002.

[11] T. W. ChUng, ET AL., Architectural and Engineering Issues for Building an Optical Internet, CANARIE Inc.

http://www. canarie.ca/ bstarn/opticalinternet.html

[12] B. Rajagopalan, J. LuCIANI, D. AwDUChE, IP over Optical Networks: A Framework, RFC 3717, March 2004.

[13] V. AlwaYn, Optical Network Design and Implementation, Cisco Press, 2004.

Received: October, 2005 Accepted: September, 2006

Contact addresses: Sudip Misra Department of Computer Science Yale University CT, USA

Subhas Chandra Misra SuperNet Solutions Toronto, ON Canada

Isaac Woungang School of Computer Science Ryerson University Toronto, ON Canada
SUDIP MISRA is a Visiting Scientist at the School of Electrical and Computer Engineering at Cornell University in Ithaca, New York, USA, and an Adjunct Professor at Ryerson University in Toronto, Canada. He received his doctoral degree from Carleton University in Ottawa, Canada. Prior to this, he received his Masters and Bachelors Degrees respectively from the University of New Brunswick in Fredericton, Canada, and the Indian Institute of Technology in Kharagpur, India. He has several years of experience working in academia, government, and private sectors. Dr. Misra has worked on several R\&D projects in project management, architecture, software design, and product engineering roles at Nortel Networks (Ottawa, Canada), Atreus Systems Corporation (Ottawa, Canada), and the Government of Ontario (Toronto, Canada).

His current research interests include network design and modeling and algorithm design and experimentation for high-performance, and highspeed telecommunication networks. His other research interests include computational learning and software engineering. He has published several research papers in journals and proceedings of international conferences, and has also received a few research awards.

SubHas ChANDRA MisRa is a Senior Project Advisor in Super Net Solutions, Scarborough, Ontario, Canada. Prior to this, he worked in the research wings of several reputed industries, such as Nortel Networks, Ottawa, Canada, and the Indian Telephone Industries, Mankapur, India. He has several years of experience working on R\&D projects in software engineering, project management, quality engineering, risk management, and project management. He has published 11 technical papers in different international journals, and 16 papers in refereed conference proceedings. He has also offered several tutorials in the allied areas. Subhas received over 15 prestigious academic awards that include Best Paper Award for one of his published papers. He has been invited as Session Chairperson in various international conferences held in USA. He is also serving as the Program Committee Member of ICEIS 2006. He received his M.Tech. degree in Computer Science and Data Processing from the Indian Institute of Technology (IIT), Kharagpur, India, and M.S. degree in Computer Science from the University of New Brunswick, Fredericton, Canada. He is also completing his $\mathrm{PhD}$ studies in Information Systems at the Eric Sprott School of Business, Carleton University, Canada.

ISAAC WoungAng received his M.A.Sc and Ph.D degrees, all in Applied Mathematics, from the Université du Sud, Toulon-Var, France, in 1990 and 1994 respectively. In 1999, he received a M.A.Sc degree from INRS-Materials and Telecommunications, University of Quebec, Canada. From 1999 to 2002, he worked as a software engineer at Nortel. Since 2002, he has been with Ryerson University, where he is now an assistant professor of computer science. In 2004, he co-founded DABNEL (the Distributed Applications and Broadband NEtworks Laboratory) R\&D group. His research interests are telecommunications network design, network security, and distributed systems. 